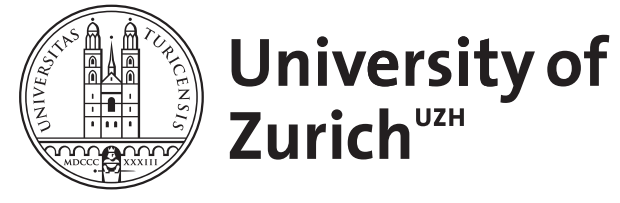

\title{
Midfacial pain
}

Lukic, Nenad ; Ettlin, Dominik

\begin{abstract}
Midfacial pain is not a diagnosis per se but rather describes an anatomical region of pain manifestation of various origins. The midface is built of cavities (nose, sinuses), calcified structures (teeth, bone) and soft tissues (nerves, muscles, mucosa, salivary glands, cartilage, skin). Since midfacial bones form the nose, sinuses and the alveolar ridge containing the upper teeth, the following three specialties are commonly consulted by patients experiencing midfacial pain
\end{abstract}

DOI: https://doi.org/10.1111/ors.12483

Posted at the Zurich Open Repository and Archive, University of Zurich

ZORA URL: https://doi.org/10.5167/uzh-187743

Journal Article

Submitted Version

Originally published at:

Lukic, Nenad; Ettlin, Dominik (2020). Midfacial pain. Oral Surgery, 13(4):415-421.

DOI: https://doi.org/10.1111/ors.12483 


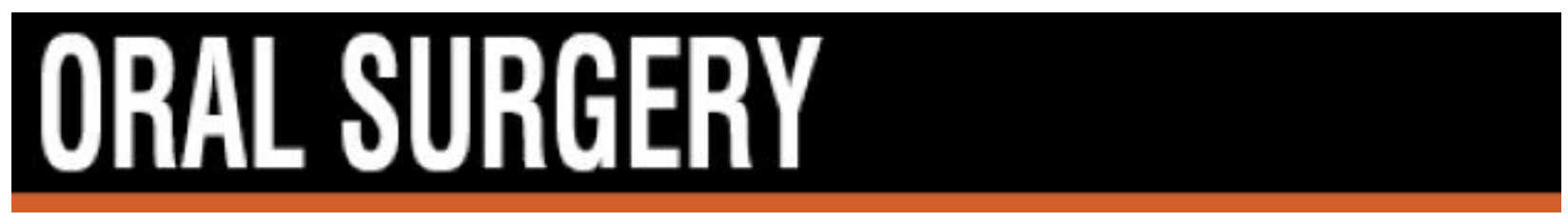

\section{Midfacial pain}

\begin{tabular}{|r|l|}
\hline Journal: & Oral Surgery \\
\hline Manuscript ID & ORS-10-19-IR-1498.R1 \\
\hline Manuscript Type: & Invited Review \\
\hline Date Submitted by the \\
Author: & n/a \\
\hline Complete List of Authors: & $\begin{array}{l}\text { Lukic, Nenad; University of Zurich, Dental Medicin } \\
\text { Ettlin, Dominik }\end{array}$ \\
\hline Keywords: & Facial Pain, Diagnosis, Headache < Facial Pain \\
\hline & \\
\hline
\end{tabular}

\section{SCHOLARONE ${ }^{m}$ \\ Manuscripts}




\section{Midfacial Pain}

Nenad Lukic, Dominik Ettlin

\section{Corresponding Author}

Dr. med., Dr. med. Dent. Nenad Lukic

University of Zurich

Center of Dental Medicine

Clinic of Masticatory Disorders

Plattenstrasse 11, 8032 Zurich

E-Mail: nenad.lukic@zzm.uzh.ch

Phone: +4144634 3395

Fax: +41446344302

Number of words (introduction to conclusion): 2460 


\section{Introduction / Background}

Midfacial pain is not a diagnosis per se but rather describes an anatomical region of pain manifestation of various origins. The midface is built of cavities (nose, sinuses), calcified structures (teeth, bone) and soft tissues (nerves, muscles, mucosa, salivary glands, cartilage, skin). Since midfacial bones form the nose, sinuses and the alveolar ridge containing the upper teeth, the following three specialties are commonly consulted by patients experiencing midfacial pain: dentists, maxillofacial surgeons, as well as ear, nose and throat (ENT) specialists. The exciting challenge for clinicians - called diagnostic process - is to match the patients' complaints in combination with clinical, imaging and/or laboratory findings to sets of diagnostic criteria defined by expert committees. Clinicians diagnosing midfacial pain need to be aware of the following:

- Mucosal alterations readily visible on imaging rarely explain pain complaints, as these generally evoke minimal or no pain (e.g. seasonal allergies).

- Discreet nerve or muscle alterations invisible on imaging may evoke disabling pain.

- Neural sensitization processes frequently blur the clinical picture.

- Primary headaches can present as midfacial pain.

- Cranial artery obstructions may present as midfacial pain.

Consequently, although a broad spectrum of midfacial pain can be confirmed by imaging, a large proportion relies solely on history taking and palpation. Cautious interpretation of image findings cannot be overemphasized. Irreversible dental or surgical treatment is justified only when a documented pathology explains the midfacial pain.

\section{Diagnostic classification of orofacial pain - a project in progress}

Diagnostic criteria for facial pain were established and repeatedly revised by the International Headache Society (IHS). The current third version of the International Classification of Headache Disorders (ICHD) is available online: www.ichd-3.org. Yet, additional nomenclature emerged in parallel, so that no uniform classification exists at this point in time. Efforts are ongoing to establish a consensus based International Classification for Orofacial Pain (ICOP). The international group working on this project is composed of experts from the IHS, the American Academy of Orofacial Pain (AAOP), the International Network for Orofacial Pain and Related Disorders 
Methodology (INfORM) and the International Association for the Study of Pain (IASP) [1]. The current Version 1.0 beta is available on www.ihs-headache.org.

\section{Differential diagnosis form a pragmatic clinical perspective}

Main features of pain are its location, quality, intensity, time pattern, triggers, modulators, and accompanying symptoms. Most commonly, the nociceptive signal is generated in facial structures where the pain is felt. Confusingly however, even when nociceptive signaling signal originates in more centrally located tissues, the pain is still experienced in peripheral structures. The mnemonic "DAMN-ODD" may serve for remembering some of the more common causes in the form of a differential diagnosis. It is briefly described in Table 1 and in more detail in Table 2. The first letter "D" stands for dental pain, which may result from pulpal or periodontal pathology. The entities arthralgia ("A") of the temporomandibular joint and myalgia ("M") of masticatory muscles are commonly grouped under the non-specific umbrella term temporomandibular disorders. Neuropathic pain ("N") may be due to a pathology located peripherally or along the primary afferent neuron (e.g. neuroma), or alternatively due to a central nervous system dysfunction (e.g. primary headache). Obstructions ("O") of arteries may lead to pain as a result of tissue hypoxia, whereas sialolithiasis and sinus obstructions cause pain by tissue expansion and/or neural irritation. Distension ("D") may further be caused by various benign or malignant tumors. Due to the anatomical complexity of the midface region and the broad differential diagnoses, inappropriate invasive procedures by doctors ("D") may induce sensitisation processes that change the original pain phenotype.

\section{Table 1}

Table 2

Next, we discuss the following entities that are not addressed by other authors, namely midface pain caused by migraine, cluster headache, nose and sinus disorders, and cardiovascular disorders.

\section{Migraine with midface pain}

Etiology and pathophysiology 
Migraine is the most common disabling headache disorder. The global prevalence is $10 \%$ in men and $22 \%$ in women [2]. Peak prevalence increases to the age of 40 years and declines after in both genders [3].

Migraine is defined as a recurrent headache disorder manifesting in attacks lasting 4-72 hours. The postulated pain mechanism includes neurogenic inflammation within the trigeminovascular system and possible links to metabolic factors $[4,5]$. Neuropeptides such as CGRP, Substance P, and Neurokinin A, are thought to be involved [6]. It has been hypothesized that the activation of a feed-forward neurovascular dilator mechanism is functionally specific for the first (ophthalmic) division of the trigeminal [7]. Clinical data indicate that this mechanism might not be restricted to the dura and the frontotemporal area, and therefore the first trigeminal branch, but may also extend to the 2nd and 3rd division involving maxillary and mandibular fibers [8]. In line with the above, nociceptive nerve fibers exhibiting Substance P- and CGRP-positive immunoreactivity have been demonstrated in human dental pulp tissue [9].

\section{Presentation and diagnosis}

Migraine has two major types: migraine without and with aura. The latter is characterized by transient focal neurological symptoms that usually precede or sometimes accompany the pain. Additionally, prodromal and postdromal symptoms may occur such as hyper- or hypoactivity, depression, cravings for particular foods, repetitive yawning, fatigue and neck stiffness and/or pain (www.ichd-3.org). Typical migraine features are unilateral location, pulsating quality, moderate to severe intensity, aggravation by routine physical activity and accompanying symptoms such as nausea and/or photophobia, phonophobia, cutaneous allodynia cranial autonomic symptoms [10].

Pain location of migraine can vary inter- and intraindividually between attacks [11, 12]. Occasionally, patients report distinctive type of episodic dental pain, lasting hours to days, sometimes associated with headache episodes and accompanying symptoms comparable to migraine and sometimes even with aura [8]. Precisely because of the pain location, these patients primarily seek help from their dentist. Imaging of the affected region will show no abnormality and the neurological examination is normal, at least during pain free intervals. Due to the experience of burdening pain in peripheral tissues, patients often seek and/or risk receiving 
unnecessary dental treatment, especially during an acute pain episode. The term orofacial migraine has been proposed to classify this migraine subtype, analogous to retinal or abdominal migraine [13]. There is evidence that more severe forms of temporomandibular disorders are associated with increased headache frequency, which applies namely for migraine [14].

\section{Management}

Migraine management options include behavioral and pharmacological interventions that are best combined. Pharmacotherapy can be implemented preventively and/or therapeutically. Preventive treatment should be offered to patients who suffer several migraine days per month that cause significant disability. Regularly updated guidelines for proper migraine treatment are listed on the website of the international headache society: www.ihs-headache.org.

\section{Future directions and conclusion}

Migraines and midfacial pain share common trigeminal nerve pathways. Future investigation on facial representations of migraines will hopefully shed light on the connection between the two. Future prevalence studies on midface pain could clarify the proportion of migraines and stimulate investigations on related mechanisms.

\section{Cluster headache with midfacial pain}

\section{Etiology and pathophysiology}

Cluster headache is defined as a severe or very severe unilateral pain in the orbital, supraorbital or temporal region. It is commonly accompanied by autonomic features [10]. It has a prevalence of about $0.1 \%$, four times more common in men. The peak age of onset is between the $3 \mathrm{rd}$ and 4 th decades. Cluster headache sufferers are often smokers

\section{Presentation and diagnosis}

Pain attacks are typically severe, strictly unilateral, last 15-180 minutes and occur from once every other day to eight times a day. The pain is associated with ipsilateral conjunctival injection, lacrimation, nasal congestion, rhinorrhoea, forehead and facial sweating, miosis, ptosis and/or eyelid edema, and/or with restlessness or agitation. Patients are typically pacing around during the headache phase. Characteristic is a 
circadian rhythmicity with attacks predominantly at night and additional circannual rhythmicity with clusters of attacks in spring and/or autumn. In the episodic form, cluster headache attacks occur in periods lasting from 7 days to one year, separated by pain-free periods lasting at least 3 months. Cluster episodes usually last between 2 weeks and 3 months, yet may extend longer in the chronic form (www.ichd-3.org).

Cluster headaches have been described as presenting in the second and third branches of the trigeminal nerve as toothache and jaw pain $[15,16]$. In patients who have already been diagnosed with a cluster headache, facial involvement was reported in $14.8 \%$ [17]. In $31 \%$ of these, the pain was predominantly perceived in the midface. Pain with neurovascular characteristics in the midface includes local hypersensitivity, episodic, unilateral throbbing pain, often waking up patients from sleep. The similarity to pulpitis is obvious and may mislead to an assumed dental diagnosis and subsequent treatment with frustrating results. Patients should actively be questioned regarding for secondary symptoms such as lacrimation, facial redness, rhinorrhea, even if these symptoms are mild [16].

\section{Management}

Acute therapy approaches include high flow oxygen for 15-20 minutes and or Triptans intranasally or subcutaneously. Regularly updated guidelines for proper cluster treatment are listed on the website of the international headache society: www.ihs-headache.org.

\section{Future directions and conclusion}

Analogous to migraines with midface pain manifestation, cluster headaches and midfacial pain share common trigeminal pathways. To prevent inappropriate procedures, there is a need to disseminate information about cluster headaches among different specialists providing care in this anatomical region (e.g. neurologists, ENT, dentists).

\section{Midfacial pain attributed to nose and sinus disorders}

Etiology, pathophysiology, presentation and diagnosis

Patients with mid facial pain and predominantly sinonasal complaints like rhinorrhea, nasal congestion, sinus pressure or pain are usually referred to an otolaryngologist. Pain presumably attributed to a diagnosis of rhinosinusitis doesn't seem to be as 
prevalent as assumed. More than $80 \%$ of patients with purulent secretions have no facial pain $[18,19]$. Patients with nasal polyposis do not complain about facial pain $[20,21]$. Patients with "only" midfacial pain receiving endoscopic sinus surgery risk to have symptoms that persist postoperatively $[22,23]$. Studies on selected facial pain patients that had a normal computed tomography and nasal endoscopy identified migraine as the most common cause of pain [24]. Also, many epidemiological studies revealed migraine as the most common cause for so called "sinus headaches". $42 \%$ of patients experiencing migraine type pain in the midface are misdiagnosed with rhinosinusitis [25], likely, because symptoms of migraine and rhinosinusitis overlap [26, 27]. In self-diagnosed or physician diagnosed "sinus headache" $86 \%$ suffered from some migraine subtype [28]. Conversely, in nonotorhinolaryngological settings, studies revealed that migraine pain is frequently misdiagnosed as sinusitis [29]. According to radiological signs, $28.7 \%$ of 1,235 patients diagnosed with a primary headache showed radiological signs of rhinosinusitis, septal spur, concha bullosa, isolated sphenoid lesion or osteoma [30]. Conversely, individuals with chronic rhinosinusitis had a 9-fold increased risk of suffering from a chronic headache. Allergic rhinitis was observed in $54 \%$ of patients with migraine [28]. It has been hypothesized that rhinosinusitis increases the risk of comorbid migraine via irritation of trigeminal nerve receptors [31].

\section{Management}

Proper diagnosis is the key to proper management of nose and sinus disorders.

\section{Future directions and conclusion}

In conclusion, rhinosinusitis rarely presents with facial pain. Research is needed that elucidates the reasons why inflammation of the maxillary sinuses is generally painless. When in doubt, observe the therapeutic principle of do no harm.

\section{Midfacial pain attributed to cardiovascular disorders}

Giant cell arteritis (GCA):

\section{Etiology and pathophysiology}

GCA is a T-cell-dependent autoimmune disease with vasculitis and genetic predisposition that affects medium and large arteries. It comprises overlapping 
phenotypes of cranial arteritis and extracranial GCA. Infections are discussed in triggering a disease outbreak, in particular viruses (e.g. HBV, influenza viruses and VZV) and bacterias (e.g. Borrelia, Klebsiella) [32]. There is a close connection with polymyalgia rheumatica.

\section{Presentation and diagnosis}

GCA should be suspected in patients older than 50 years who experience unilateral or bilateral jaw muscle tenderness, jaw claudication, visual disturbances, and tenderness in the area of the temporal arteries [33]. Elevated erythrocyte sedimentation rate $(>50 / \mathrm{h}$ ) and C-reactive protein (>50 $\mathrm{mg} / \mathrm{L})$ increases the probability of GCA. Biopsy of the GCA is the gold standard diagnostic test and should be considered when GCA is suspected. Imaging can also aid in the diagnosis, namely duplex sonography, high-resolution magnetic resonance imaging, and positron-emission tomography [33, 34].

\section{Management}

GCA is a medical emergency. High doses of corticosteroids (40-60 mg) are indicated when there is an urgent suspicion of the disease. In case of spread to ocular or cerebral vessels (threat of blindness or life-threatening stroke) $500 \mathrm{mg}$ $1 \mathrm{~g}$ of methylprednisolone applied intravenously three times daily are indicated. Biological agents are effective and safe corticosteroid-sparing agents in treating GCA.

Carotid artery dissection (CAD):

\section{Etiology and pathophysiology}

CAD can occur spontaneously or as a result of trauma or defect with intimal wall damage. The splitting of the arterial wall forms a new lumen with secondary thrombosis, which can lead to pseudoaneurysm, vessel occlusion, embolisms with the risk of consecutive cerebral apoplexy. The carotid wall is innervated by the trigeminal nerve so the pain is attributed to a stimulation of the trigeminovascular system.

\section{Presentation and diagnosis}

Due to new imaging techniques we have evidence, that CAD and vertebral artery dissections show an annual incidence rate of 5 per 100,000 [35]. 95\% of the patients 
describe new unilateral steady or throbbing pain in the head, face, eye, neck, and toothache ipsilateral to the defect [35-37]. A Horner's Syndrome may be present if adjacent sympathetic nerve fibers are compressed. An ischemic stroke due to CAD may manifest with disturbance of consciousness (e.g. confusion) and severe focal neurological signs (e.g. unilateral numbness or facial weakness, difficulties to speak or understand, or to see on or both eyes, as well as loss of coordination).

\section{Management}

Timely and appropriate diagnostic strategies permlay the ground for early and effective treatment strategies [38].

\section{Conclusion}

When a patient presents with stroke symptoms in combination with a severe orofacial pain, a carotid artery dissection or similar vascular conditions should be suspected [10].

\section{Ischemic heart disease (IHD)}

\section{Etiology and pathophysiology}

IHD accounts for one third of all deaths annually, and approximately 2-3\% of acute myocardial infarctions are missed in the emergency department [39, 40]. Pain manifestation in the orofacial region are thought to be mediated by afferent fibers of the vagus nerve transmitting nociceptive information to cervical neurons [41-43].

\section{Presentation and diagnosis}

Pain due to IHD typically refers to the shoulder or arm. Yet, it can also affect the face, jaw and ear [44, 45]. A systematic review indicated that midface pain was the sole symptom of cardiac ischemia in approximately $6 \%$ of cases [46, 47]. Patients often described their midface pain as "pressure" or "burning". The following situation should trigger a screening electrocardiogram: bilateral midfacial pain that is evoked by exercise, or associated with chest discomfort, shortness of breath, nausea or fatigue [48].

\section{Management}

Management ought to be directed towards the underlying cause. 


\section{Conclusion}

Midface pain due to IHD carries the risk of misdirected dental treatment and more importantly, delay of urgent medical care. 


\section{Table 1}

The mnemonic of midfacial pain etiologies "DAMN-ODD" serves the dentist or specialist for remembering the directions of the more common causes in the diagnostic process.

\section{Table 2}

Midfacial pain diagnosis grouped by the mnemonic DAMN-ODD with ICHD-3 classification codes and the referral to topics discussed in this issue by other authors. 


\section{References}

1 Benoliel R. Editorial: Let's Adopt ICOP: Speaking the Same Language. J Oral Facial Pain Headache 2019; 33 (3): 245-246

2 Stovner L, Hagen $K$, Jensen $R$ et al. The global burden of headache: A documentation of headache prevalence and disability worldwide.

Cephalalgia 2007; 27 (3): 193-210

3 Lipton RB, Silberstein SD, Stewart WF. An update on the epidemiology of migraine. Headache 1994; 34 (6): 319-328

4 Bolay $H$, Reuter $U$, Dunn AK et al. Intrinsic brain activity triggers trigeminal meningeal afferents in a migraine model. Nat Med 2002; 8 (2): 136-142

5 Gross EC, Lisicki M, Fischer D et al. The metabolic face of migraine - from pathophysiology to treatment. Nat Rev Neurol; DOI: 10.1038/s41582-0190255-4

6 May A, Büchel C, Turner $R$ et al. Magnetic resonance angiography in facial and other pain: Neurovascular mechanisms of trigeminal sensation. $J$ Cereb Blood Flow Metab 2001; 21 (10): 1171-1176

7 Rodd HD, Boissonade FM. Comparative immunohistochemical analysis of the peptidergic innervation of human primary and permanent tooth pulp. Arch Oral Biol 2002; 47 (5): 375-385

8 Gaul C, Sándor PS, Galli U et al. Orofacial migraine. Cephalalgia 2007; 27 (8): 950-952

9 Hargreaves $K M$, Bowles WR, Jackson DL. Intrinsic regulation of CGRP release by dental pulp sympathetic fibers. J Dent Res 2003; 82 (5): 398-401

10 Headache Classification Committee of the International Headache Society (IHS) The International Classification of Headache Disorders, 3rd edition. Cephalalgia 2018; 38 (1): 1-211

11 Kelman L. Migraine pain location: A tertiary care study of 1283 migraineurs. Headache 2005; 45 (8): 1038-1047

12 Hussain A, Stiles MA, Oshinsky ML. Pain remapping in migraine: A novel characteristic following trigeminal nerve injury. Headache 2010; 50 (4): 669671

13 Gaul C, Sándor PS, Ettlin DA. Neurovascular orofacial pain: Authors' reply. Cephalalgia 2008; 28 (3): 305

14 Gonçalves DAG, Camparis CM, Speciali JG et al. Temporomandibular disorders are differentially associated with headache diagnoses: A controlled study. Clin J Pain 2011; 27 (7): 611-615

15 Gaul C, Gantenbein AR, Buettner UW et al. Orofacial cluster headache. Cephalalgia 2008; 28 (8): 903-905

16 Benoliel R, Sharav Y, Eliav E. Neurovascular orofacial pain. J Am Dent Assoc 2010; 141 (9): 1094-1096

17 Ziegeler C, May A. Facial presentations of migraine, TACs, and other paroxysmal facial pain syndromes. Neurology 2019; 93 (12): e1138-e1147

18 West B, Jones NS. Endoscopy-negative, computed tomography-negative facial pain in a nasal clinic. Laryngoscope 2001; 111 (4 Pt 1): 581-586

19 Walliczek-Dworschak $U$, Diogo I, Strack $L$ et al. Indicazioni all'esecuzione di una TC cone beam del distretto testa e collo nei bambini. Acta Otorhinolaryngol Ital 2017; 37 (4): 270-275

20 Fahy C, Jones NS. Nasal polyposis and facial pain. Clin Otolaryngol Allied Sci 2001; 26 (6): 510-513 
21 Gelardi M, lannuzzi $L$, Giosa $M$ de et al. Trattamento medico della rinosinusite cronica con poliposi naso-sinusale sulla base del sistema di grading clinico-citologico per un approccio medico personalizzato. Acta Otorhinolaryngol Ital 2017; 37 (1): 38-45

22 Tarabichi M. Characteristics of sinus-related pain. Otolaryngol Head Neck Surg 2000; 122 (6): 842-847

23 Jones NS, Cooney TR. Facial pain and sinonasal surgery. Rhinology 2003; 41 (4): 193-200

24 Perry BF, Login IS, Kountakis SE. Nonrhinologic headache in a tertiary rhinology practice. Otolaryngol Head Neck Surg 2004; 130 (4): 449-452

25 Graff-Radford SB. Facial pain. Curr Opin Neurol 2000; 13 (3): 291-296

26 Barbanti $P$, Fabbrini G, Pesare $M$ et al. Unilateral cranial autonomic symptoms in migraine. Cephalalgia 2002; 22 (4): 256-259

27 Schreiber CP, Hutchinson S, Webster CJ et al. Prevalence of migraine in patients with a history of self-reported or physician-diagnosed "sinus" headache. Arch Intern Med 2004; 164 (16): 1769-1772

28 Eross E, Dodick D, Eross M. The Sinus, Allergy and Migraine Study (SAMS). Headache 2007; 47 (2): 213-224

29 Lipton RB, Diamond S, Reed $M$ et al. Migraine diagnosis and treatment: Results from the American Migraine Study II. Headache 2001; 41 (7): 638645

30 Lee J-H, Kim H-J, Hong $\mathrm{Y}-\mathrm{H}$ et al. Underestimation of Rhinogenic Causes in Patients Presenting to the Emergency Department with Acute Headache. Acta Neurol Taiwan 2015; 24 (2): 37-42

31 Aaseth K, Grande RB, Kvaerner K et al. Chronic rhinosinusitis gives a ninefold increased risk of chronic headache. The Akershus study of chronic headache. Cephalalgia 2010; 30 (2): 152-160

32 Lavi E, Gilden D, Nagel M et al. Prevalence and distribution of VZV in temporal arteries of patients with giant cell arteritis. Neurology 2015; 85 (21): 1914-1915

33 Veldhoen S, Klink T, Geiger J et al. MRI displays involvement of the temporalis muscle and the deep temporal artery in patients with giant cell arteritis. Eur Radiol 2014; 24 (11): 2971-2979

34 Schmidt WA. Ultrasound in the diagnosis and management of giant cell arteritis. Rheumatology (Oxford) 2018; 57 (suppl_2): ii22-ii31

35 Mokri B. Headaches in cervical artery dissections. Curr Pain Headache Rep 2002; 6 (3): 209-216

36 Biousse V MP. Carotid or vertebral artery disease. In: Carotid or vertebral artery disease., Hrsg.: Carotid or vertebral artery disease. Philadelphia:: LippincottWilliams andWilkins; 2006: 911-918

37 Abbott JJ, Newman AC, Schievink WI. Maxillary tooth pain as a symptom of internal carotid artery dissection: Case series. J Am Dent Assoc 2017; 148 (6): 399-403

38 Benoliel $R$, Svensson $P$, Evers $S$ et al. The IASP classification of chronic pain for ICD-11: Chronic secondary headache or orofacial pain. Pain 2019; 160 (1): 60-68

39 Capewell S, Ford ES, Croft JB et al. Cardiovascular risk factor trends and potential for reducing coronary heart disease mortality in the United States of America. Bull World Health Organ 2010; 88 (2): 120-130 
40 Pope JH, Aufderheide TP, Ruthazer $R$ et al. Missed diagnoses of acute cardiac ischemia in the emergency department. N Engl J Med 2000; 342 (16): 1163-1170

41 Foreman RD. Neurological mechanisms of chest pain and cardiac disease. Cleve Clin J Med 2007; 74 Suppl 1: S30-3

42 Kreiner $M$, Okeson J, Tanco $V$ et al. Orofacial Pain and Toothache as the Sole Symptom of an Acute Myocardial Infarction Entails a Major Risk of Misdiagnosis and Death. J Oral Facial Pain Headache; DOI: 10.11607/ofph.2480

43 Kreiner M, Alvarez $R$, Waldenstrom A et al. Craniofacial pain of cardiac origin is associated with inferior wall ischemia. J Oral Facial Pain Headache 2014; 28 (4): 317-321

44 Myers $D E$. Toothache referred from heart disease and lung cancer via the vagus nerve. Gen Dent 2010; 58 (1): e2-5

45 Amirhaeri S, Spencer $D$. Myocardial infarction with unusual presentation of otalgia: A case report. Int J Emerg Med 2010; 3 (4): 459-460

46 Jalali N, Vilke GM, Korenevsky M et al. The tooth, the whole tooth, and nothing but the tooth: Can dental pain ever be the sole presenting symptom of a myocardial infarction? A systematic review. J Emerg Med 2014; 46 (6): 865-872

47 Bakhshi M, Rezaei R, Baharvand $M$ et al. Frequency of craniofacial pain in patients with ischemic heart disease. J Clin Exp Dent 2017; 9 (1): e91-e95

48 Benjamin EJ, Muntner P, Alonso A et al. Heart Disease and Stroke Statistics-2019 Update: A Report From the American Heart Association. Circulation 2019; 139 (10): e56-e528 


\author{
Table 1: \\ D - $\quad$ Dentogenic \\ A - $\quad$ Arthrogenic \\ M - Myogenic \\ N - $\quad$ Neurogenic (primary, secondary) \\ O - $\quad$ Obstruction of arteries, salivary glands, sinuses \\ D - $\quad$ Distension by tumors \\ D - $\quad$ Doctor induced (sensitisation processes)
}


Table 2:

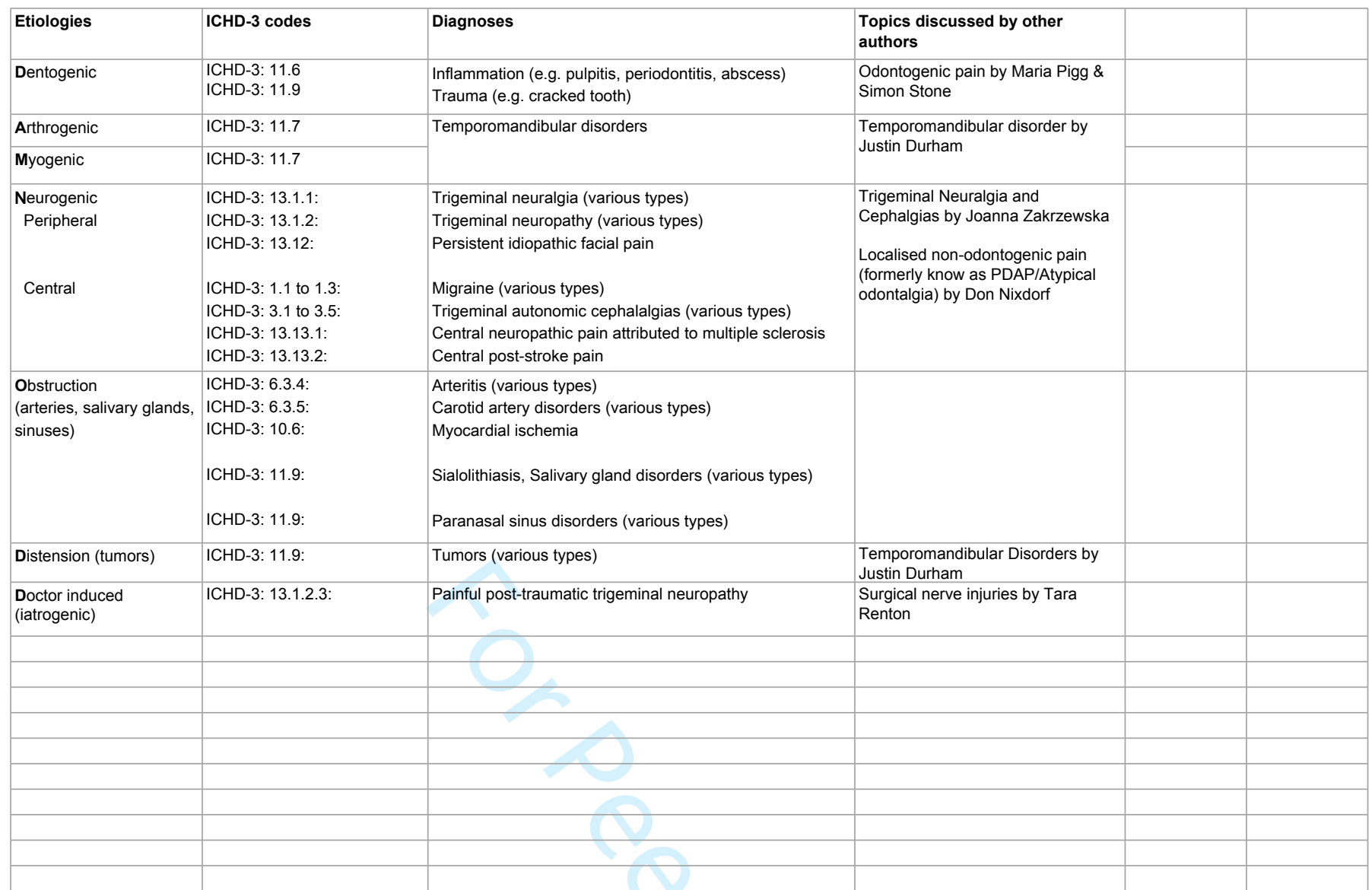

